\title{
Tensorial generalized Stokes-Einstein relation for anisotropic probe microrheology
}

\author{
Todd M. Squires • Thomas G. Mason
}

Received: 12 January 2010 / Revised: 30 July 2010 / Accepted: 7 September 2010 / Published online: 26 September 2010

(C) The Author(s) 2010. This article is published with open access at Springerlink.com

\begin{abstract}
In thermal "passive" microrheology, the random Brownian motion of anisotropically shaped probe particles embedded within an isotropic viscoelastic material can be used to extract the material's frequencydependent linear viscoelastic modulus. We unite the existing theoretical frameworks for separately treating translational and rotational probe motion in a viscoelastic material by extending the generalized Stokes-Einstein relation (GSER) into a tensorial form that reflects simultaneous equilibrium translational and rotational fluctuations of one or more anisotropic probe particles experiencing viscoelastic drag. The tensorial GSER provides a formal basis for interpreting the complex Brownian motion of anisotropic probes in a viscoelastic material. Based on known hydrodynamic calculations of the Stokes mobility of highly symmetric shapes in a simple viscous liquid, we show simple examples of the tensorial GSER for spheroids and half-stick, half-slip Janus spheres.
\end{abstract}

Keywords Microrheology • Brownian dynamics • Colloids $\cdot$ Linear viscoelasticity $\cdot$ Stokes-Einstein relation - Anisotropic particle

\section{T. M. Squires}

Department of Chemical Engineering,

University of California, Santa Barbara,

CA 93106-5080, USA

T. G. Mason $(\varangle)$

Department of Physics and Astronomy,

Department of Chemistry and Biochemistry, and California NanoSystems Institute,

University of California, Los Angeles,

CA 90095-1569, USA

e-mail:mason@chem.ucla.edu

\section{Introduction}

Passive translational microrheology is a method that is frequently used for extracting the frequencydependent linear viscoelastic modulus from the Brownian fluctuations of a probe particle in a viscoelastic material (Mason and Weitz 1995; Gardel and Weitz 2005; Squires and Mason 2010). Typically, spherical probes are introduced into the material at a very dilute concentration and their translational fluctuations are measured independent of their rotational motion. Whether time-averaged, ensemble-averaged, or both, these fluctuations are interpreted using a generalized Stokes-Einstein relation (GSER) (Mason and Weitz 1995; Mason et al. 1997b). The Einstein part of the GSER provides a measure of local mobility or equivalently frictional drag on the probe, whereas the Stokes part of the GSER provides a means of connecting the shape-dependent mobility with the intensive property of the material's viscoelastic modulus outside the probe (Squires and Mason 2010). One main advantage of passive microrheology over conventional macroscopic rheology is the ability to access the rheological properties of exotic and expensive materials, including biomaterials such as DNA (Mason et al. 1997a), F-actin (Gittes et al. 1997), and block copolypeptides (Breedveld and Pine 2003), for which providing a quantity necessary for macroscopic rheology is typically prohibitive. Other advantages include obtaining the linear viscoelastic (LVE) shear modulus (Bird et al. 1977) over a very wide frequency range without time-temperature superposition, the ability to probe local properties of heterogeneous materials, and to make measurements of non-equilibrium glassy (Mason et al. 1997b), slowly 
evolving (Sato and Breedveld 2006), and athermally driven (Mizuno et al. 2007) systems.

Separate translational and rotational versions of the GSER have been previously developed without being combined. The first passive microrheology experiments introduced the translational GSER for spherical probes and showed its general utility through light scattering measurements (Mason and Weitz 1995). This generality of this basic approach was also demonstrated later using real-space particle tracking of spherical probes (Mason et al. 1997a; Gittes et al. 1997). Following translational studies, a rotational form of passive microrheology based on a rotational GSER (i.e., a generalized Stokes-Einstein-Debye relation) was introduced and demonstrated using thermally driven rotation of wax microdisks in a viscoelastic polymer solution (Cheng and Mason 2003); measurements of rotational fluctuations of optically anisotropic spheres in an LVE material further validated this (Bishop et al. 2004; Schmiedeberg and Stark 2005; Andablo-Reyes et al. 2005). More recently, the advent of new methods for colloidal synthesis and production, whether top-down (see e.g., Mason 2002; Hernandez and Mason 2007; Badaire et al. 2007) or bottom-up (see e.g., Manoharan et al. 2003; Champion et al. 2007), for making customshaped microscale probes provides the potential for simultaneously determining both translational and rotational dynamics from optical microscopic imaging methods. To fully exploit the information about the material's viscoelasticity contained in the fluctuating positions and angles of an anisotropic probe, it would be useful to extend these simple versions of the GSER to handle a combination of translational and rotational degrees of freedom (DOF), consistent with energy equipartition and the fluctuation-dissipation theorem (Reif 1965).

The diffusion of anisotropic particles in a simple viscous liquid is well known for a number of limiting cases. Anisotropic diffusion of non-spherical probes has been observed both through light scattering (Berne and Pecora 2000) and direct microscopic visualization (Han et al. 2006, 2009). In general, anisotropic colloidal particles experience anisotropic diffusion, both in translation and rotation, resulting from an anisotropic hydrodynamic resistance that depends upon the specific details of a particle's shape. At low enough frequencies where inertial effects can be neglected, solutions to the Stokes equations for viscous flow around an anisotropic probe particle with specified translational and rotational velocity yields both forces and torques, giving a self-resistance tensor $\zeta$ (Happel and Brenner 1983; Kim and Karilla 1991; Leal 2007) that can be related directly to anisotropic diffusivities. In other words, the instantaneous orientation of a colloidal particle, which is subjected to Brownian fluctuations in a liquid, can influence the propensity of the particle to translate and rotate in certain directions thereafter.

Here, following the derivations for the translational GSER (Mason and Weitz 1995) and the rotational GSER (Cheng and Mason 2003), we combine the two separate approaches into a unified tensorial GSER (T-GSER) capable of describing anisotropic Brownian motion of anisotropically shaped particles in a simple isotropic, incompressible, viscoelastic material. This unification effectively provides a complete set of equations that can be used to predict a material's linear viscoelastic shear modulus from all possible modes of translational and/or rotational probe motion, thereby enabling the moduli calculated from different measured degrees of freedom of probe motion to be crosschecked for self-consistency. To further illustrate this unification, we summarize known expressions of the tensorial GSER for prolate and oblate spheroids (i.e., ellipsoids of revolution), as well as axisymmetric bodies without fore-aft symmetry, for which translation and rotation are coupled. In addition, we provide a further generalization of the tensorial GSER to $N$ particles, setting the stage for multi-point translational and rotational microrheology of anisotropically shaped probes that can potentially be used to obtain more accurate results for the viscoelasticity of heterogeneous materials.

\section{Theory}

In this section, we generalize the mobility to account for linear viscoelastic media, which introduces memory into the mobility and resistance and also for probes having arbitrary shape, potentially coupling translation to rotation. By relating this generalized mobility to the translational and rotational fluctuations of a probe particle in a LVE material through energy equipartition and the fluctuation-dissipation theorem, we arrive at a tensorial expression for the GSER that accounts for both translation and rotation. Moreover, we extend this generalization even further to address arbitrary numbers of particles that interact through the LVE material between them, thereby coupling the response of two or more distinct probes, yielding a separationdependent response.

Generalized mobility and resistance for spherical probes

The hydrodynamic mobility is the linear response property of a particle in a material, and it is important in 
passive microrheology, where stochastic thermal forces drive linear response motion of colloidal probes. In the low-Reynolds number limit, the velocity of a sphere of radius $a$ forced to move through a Newtonian fluid of viscosity $\eta_{0}$ is given by $V=M_{0} F_{H}$, where $M_{0}=$ $\left(6 \pi \eta_{0} a\right)^{-1}$ is the steady Stokes mobility of a sphere. The Newtonian hydrodynamic resistance $\zeta_{0}$ is the inverse of the mobility, and gives the hydrodynamic force $F_{H}=$ $\zeta_{0} V$ required to maintain a specified sphere velocity $V(t)$. These simple scalars are valid for spheres undergoing quasi-steady motion in a Newtonian fluid.

We generalize probe mobility to account for linear viscoelastic media, following Zwanzig and Bixon (1970). The velocity of a sphere at time $t$ in response to a time-dependent, hydrodynamic force $F_{H}\left(t^{\prime}\right)$ exerted over a history of past times $t^{\prime}$ is given by

$V(t)=\int_{-\infty}^{t} M\left(t-t^{\prime}\right) F_{H}\left(t^{\prime}\right) \mathrm{d} t^{\prime}$,

where $M\left(t-t^{\prime}\right)$ is the self-mobility of the particle, generalized to have a memory corresponding to a LVE fluid. The corresponding relation giving the hydrodynamic force on a sphere with specified velocity history $V\left(t^{\prime}\right)$ is

$F_{H}(t)=-\int_{-\infty}^{t} \zeta\left(t-t^{\prime}\right) V\left(t^{\prime}\right) \mathrm{d} t^{\prime}$

where $\zeta\left(t-t^{\prime}\right)$ is the generalized self-resistance (i.e., drag) of the particle moving in the LVE material. Two features are noteworthy. Firstly, the hydrodynamic force $F_{H}(t)$ contains exclusively the viscoelastic and (possibly) inertial stresses of the fluid. Specifically, $F_{H}$ does not include the inertia of the particle. Secondly, the mobility and resistance are no longer simply inverses of each other. However, Fourier or Laplace transforms de-convolve Eqs. 1 and 2 to reveal that the transformed mobility and resistance functions are inverses of each other:

$\tilde{M}(\omega)=(\tilde{\zeta}(\omega))^{-1}$

The mobility at any given frequency-Laplace or Fourier-is thus the inverse of the resistance at that frequency.
Throughout this work, we will use one-sided (i.e., unilateral) Fourier transforms, defined by

$\mathcal{F}^{u}(g(t)) \equiv \tilde{g}(\omega)=\int_{0}^{\infty} e^{-i \omega t} f(t) \mathrm{d} t$.

Following Mason (2000), entirely analogous results can be obtained using unilateral Laplace transforms or bilateral Fourier transforms. Regardless of the specific type of transform, each provides an equivalent representation of the information contained in the timedomain function. For example, Laplace-transformed quantities can be obtained from the unilateral Fouriertransformed ones using simple analytic continuation $(s=i \omega)$. Inverse unilateral transformation from the frequency domain back to the time domain involves complex integration (Davies 2002; Roos 1969), so it is typically convenient to present simpler and more compact expressions in the frequency domain.

Generalized mobility and resistance tensors for an anisotropic probe

We now consider the equations of motion containing a generalized mobility for an anisotropic particle. Neglecting particle inertia, the translational velocity $\mathbf{V}$ and angular velocity $\boldsymbol{\Omega}$, under the influence of a force $\mathbf{F}$ and torque $\mathbf{T}$ in a viscous fluid, are given by

$\left(\begin{array}{l}\mathbf{V}(t) \\ \boldsymbol{\Omega}(t)\end{array}\right)=\int_{-\infty}^{t} \mathbf{M}\left(t-t^{\prime}\right) \cdot\left(\begin{array}{c}\mathbf{F}\left(t^{\prime}\right) \\ \mathbf{T}\left(t^{\prime}\right)\end{array}\right) \mathrm{d} t^{\prime}$

Here

$\mathbf{M}(t)=\left(\begin{array}{ll}\mathbf{M}_{\mathbf{V F}}(t) & \mathbf{M}_{\mathbf{V T}}(t) \\ \mathbf{M}_{\Omega \mathbf{F}}(t) & \mathbf{M}_{\Omega \mathbf{T}}(t)\end{array}\right)$

is the $6 \times 6$ generalized self-mobility tensor for the particle. It consists of four $3 \times 3$ sub-blocks; $\mathbf{M}_{\mathbf{V T}}$ gives the translational velocity $\mathbf{V}$ in response to a torque $\mathbf{T}$ (which would generally be nonzero for chiral particles), and the other sub-blocks are defined in a similar manner. The generalized self-resistance tensor $\zeta(t)$ is the response function analogous to the mobility tensor that describes the forces and torques on a probe resulting from its past history of translational and rotational velocities:

$\left(\begin{array}{l}\mathbf{F}(t) \\ \mathbf{T}(t)\end{array}\right)=\int_{-\infty}^{t} \zeta\left(t-t^{\prime}\right) \cdot\left(\begin{array}{l}\mathbf{V}\left(t^{\prime}\right) \\ \mathbf{\Omega}\left(t^{\prime}\right)\end{array}\right) \mathrm{d} t^{\prime}$.

As in the scalar case, the mobility tensor is most easily related to the resistance tensor through transformation 
of the two equations above, since the convolution integrals become products in Fourier space: the transformed resistance and transformed mobility tensors are simply inverses of each other:

$\tilde{\mathbf{M}}(\omega) \cdot \tilde{\zeta}(\omega)=\delta$, so $\tilde{\mathbf{M}}=(\tilde{\zeta})^{-1}$

Here, $\delta$ is the unity tensor. In the limiting case of a Newtonian fluid undergoing Stokes flow around an anisotropic probe, neither tensor depends on frequency, and the Stokes mobility tensor can be simply obtained from matrix inversion of the Stokes resistance tensor.

Single-particle tensorial GSER: Einstein component

In this section, we treat a single anisotropic particle in an isotropic LVE material, and derive the Einstein component of a tensorial GSER that provides a means for obtaining the probe's frequency-dependent selfmobility tensor, which reflects a combination of the probe particle's geometry and the material's viscoelasticity. We relate the average statistical properties of a probe's translational and rotational motion to the selfmobility tensor using the energy equipartition theorem and the fluctuation-dissipation theorem (FDT).

The entire set of translational and rotational equations of motion for a single particle in an isotropic LVE material can be captured by a tensorial version of the generalized Langevin equation:

$\mathbb{M} \cdot \dot{\mathcal{V}}(t)=\mathcal{F}_{R}(t)-\int_{-\infty}^{t} \zeta\left(t-t^{\prime}\right) \cdot \mathcal{V}\left(t^{\prime}\right) \mathrm{d} t^{\prime}$

where $\mathbb{M}$ is a $6 \times 6$ matrix giving mass and moments of inertia, $\mathcal{F}_{R}=\left\{\mathbf{f}_{R}, \mathbf{T}_{R}\right\}$ combines random forces and torques into a single six-component vector, $\mathcal{V}=\{\mathbf{V}, \boldsymbol{\Omega}\}$ combines translational and rotational velocities into a six-component vector, and $\zeta$ is a $6 \times 6$ generalized resistance tensor that reflects both particle geometry as well as the linear viscoelastic memory of the material. Taking the unilateral Fourier transform of Eq. 9, and solving for the $k$ th scalar component of $\tilde{\mathcal{V}}(\omega)$, one obtains:

$\tilde{\mathcal{V}}_{k}(\omega)=(i \mathbb{M} \omega+\tilde{\zeta}(\omega))_{k \ell}^{-1} \cdot\left(\tilde{\mathcal{F}}_{R}(\omega)+\mathbb{M} \cdot \mathcal{V}(0)\right)_{\ell}$,

where we use the Einstein indexing convention, implying summation over $\ell$. Multiplying Eq. 10 by $\mathcal{V}_{j}(0)$ and ensemble averaging both sides (denoted $\langle\cdot\rangle$ ) gives the transformed velocity correlation function (VCF) between components $j$ and $k$ of the generalized velocities:

$$
\begin{aligned}
& \left\langle\mathcal{V}_{j}(0) \tilde{\mathcal{V}}_{k}(\omega)\right\rangle \\
& =(i \mathbb{M} \omega+\tilde{\zeta}(\omega))_{k \ell}^{-1} \\
& \quad \cdot\left(\left\langle\mathcal{V}_{j}(0) \tilde{\mathcal{F}}_{R, \ell}(\omega)\right\rangle+\left\langle\mathcal{V}_{j}(0)(\mathbb{M} \cdot \mathcal{V}(0))_{\ell}\right\rangle\right)
\end{aligned}
$$

We now employ two key facts from equilibrium statistical mechanics (see e.g., Reif 1965; Landau and Lifshitz 2000). Firstly, the stochastic thermal force $\mathcal{F}_{R}$ is uncorrelated with any velocity component of the probe, so $\left\langle\mathcal{V}_{j} \tilde{\mathcal{F}}_{R, \ell}\right\rangle=0$. Secondly, the energy equipartition theorem states that each independent quadratic degree of freedom has an average of $\frac{1}{2} k_{B} T$ of energy, so that $\left\langle\mathcal{V}_{j}(0)(\mathbb{M} \cdot \mathcal{V}(0))_{\ell}\right\rangle=k_{B} T \delta_{j \ell}$, yielding the following expression for the VCF after summation:

$\left\langle\mathcal{V}_{j}(0) \tilde{\mathcal{V}}_{k}(\omega)\right\rangle=k_{B} T(i \mathbb{M} \omega+\tilde{\zeta}(\omega))_{j k}^{-1}$,

where the inverse operation is performed before indexing. Most microrheology experiments are performed at frequencies low enough that the LVE resistance $|\tilde{\zeta}(\omega)|$ dominates over probe inertia $|\mathbb{M} \omega|$. In this resistancedominated limit, which may still extend up to frequencies even beyond the $\mathrm{MHz}$ range for microscale or smaller probes, we can ignore the probe inertia, and the transformed VCF is simply $k_{B} T$ times the corresponding element of the mobility tensor:

$\left\langle\mathcal{V}_{j}(0) \tilde{\mathcal{V}}_{k}(\omega)\right\rangle=k_{B} T(\tilde{\zeta}(\omega))_{j k}^{-1}=k_{B} T(\tilde{\mathbf{M}}(\omega))_{j k}$.

This relationship simply and effectively expresses the fluctuation-dissipation theorem that connects the probe's generalized mobility to the statistical average properties of its motion in thermal equilibrium through its generalized VCF, taking into account all independent degrees of its translational and rotational freedom. Equation 13 is perhaps the simplest and most direct expression of the Einstein component of the GSER in the non-inertial limit.

In the time domain, each VCF can be related to a corresponding mean square displacement (MSD) of generalized displacement components $\Delta \mathcal{R}_{j}$ and $\Delta \mathcal{R}_{k}$ 
(i.e., where the six-component generalized position vector is $\mathcal{R}=\{\mathbf{r}, \boldsymbol{\theta}\})$ by:

$\left\langle\mathcal{V}_{j}(0) \mathcal{V}_{k}(t)\right\rangle=\frac{1}{2} \frac{d^{2}}{d t^{2}}\left\langle\Delta \mathcal{R}_{j}(0) \Delta \mathcal{R}_{k}(t)\right\rangle$

or equivalently in the Fourier domain as

$$
\left\langle\mathcal{V}_{j}(0) \tilde{\mathcal{V}}_{k}(\omega)\right\rangle=\frac{(i \omega)^{2}}{2} \mathcal{F}^{u}\left\langle\Delta \mathcal{R}_{j}(0) \Delta \mathcal{R}_{k}(t)\right\rangle
$$

This general VCF relationship expresses both velocity autocorrelation (VAC) functions, as well as velocity cross-correlation functions, in terms of self- and coupledtranslational and rotational MSDs, respectively.

The general version of the Einstein component of the GSER, expressed in terms of MSDs in the unilateral Fourier domain, then follows:

$$
\begin{aligned}
\mathcal{F}^{u}\left(\Delta \mathcal{R}_{j}(0) \Delta \mathcal{R}_{k}(t)\right) & =\left\langle\Delta \mathcal{R}_{j}(0) \Delta \tilde{\mathcal{R}}_{k}(\omega)\right\rangle \\
& =\frac{2 k_{B} T}{(i \omega)^{2}}(\tilde{\mathbf{M}}(\omega))_{j k} .
\end{aligned}
$$

The above equation is another statement of the FDT that is entirely equivalent to Eq. 13. Moreover, alternative expressions for both Eqs. 13 and 16 can be written in the unilateral Laplace domain by replacing $i \omega$ with the Laplace frequency $s$ and functional dependencies $(\omega)$ with $(s)$.

Single-particle tensorial GSER: Stokes component

In general, it is not possible to extract a material's LVE response given only the Einstein component of the GSER, because the generalized mobility tensor depends upon both probe characteristics and LVE material properties. Under certain conditions, however, the (Newtonian) Stokes flow solutions around a moving probe can be generalized to hold for LVE materials at all frequencies (Schnurr et al. 1997; Squires, in preparation) assuming these conditions to hold for a given material, knowing the Stokes resistance or mobility of a probe enables the material's LVE properties to be extracted from one or more elements of the measured VAC or MSD. Thus, thermally driven fluctuations involving different degrees of freedom of the probe particle can provide the same intensive equilibrium property (frequency-dependent shear modulus) as would be measured macroscopically. Knowing the Stokes flow solutions for the motion of a particular particle shape enables the linear viscoelastic response of a given material to be determined independently from any of the independent degrees of freedom of the probe motion. Having multiple expressions (and measurements), all of which should yield the same LVE modulus, enables measurements involving different degrees of freedom to be cross-checked for consistency, or combined for better statistics and greater precision.

The generalized Stokes mobility (GSM) assumes that the probe's mobility $\tilde{\mathbf{M}}(\omega)$ and, equivalently, its resistance $\tilde{\zeta}(\omega)$, in the LVE material are given precisely by the Stokes solutions in a Newtonian fluid, where the Newtonian viscosity $\eta_{0}$ is effectively replaced by the material's frequency-dependent complex viscosity $\tilde{\eta}(\omega)$ :

$\tilde{\mathbf{M}}(\omega)=\frac{\eta_{0}}{\tilde{\eta}(\omega)} \mathbf{M}_{\mathrm{St}}$,

where $\mathbf{M}_{\mathrm{St}}$ is the $6 \times 6$ frequency-independent Stokes mobility tensor. Since each element in $\mathbf{M}_{\mathrm{St}}$ is inversely proportional to $\eta_{0}$, one can define a rescaled Stokes mobility tensor:

$\mathbf{M}_{\mathrm{St}}^{\mathrm{r}}=\eta_{0} \mathbf{M}_{\mathrm{St}}$,

which is independent of the Newtonian viscosity $\eta_{0}$ and depends only on the assumed particle size, shape, and boundary conditions. Thus, a simplified expression for the GSM is

$\tilde{\mathbf{M}}(\omega)=\frac{\mathbf{M}_{\mathrm{St}}^{\mathrm{r}}}{\tilde{\eta}(\omega)}$,

where the numerator contains size and shape parameters of the probe based on Stokes flow solutions, and the denominator contains the desired frequencydependent LVE response of the material through its complex viscosity. The simple form for the GSM in Eq. 19 is convenient because the material's complex viscosity will not depend explicitly on any assumed value for $\eta_{0}$. A systematic demonstration of the conditions under which the GSM assumption is indeed valid (a broad class of homogeneous, isotropic, incompressible, continuum LVE materials), as well as when and how the GSM can be extended when such conditions are violated, will be published separately (Squires, in preparation). 
Single-particle tensorial GSER

for an anisotropic probe

By combining the Einstein and Stokes components presented in the preceding two sections, it is possible to express a wide range of equivalent statements of the GSER for extracting the material's LVE response from the motion of a single anisotropic probe particle. For instance, by combining Eqs. 19 and 13, the complex viscosity (denoted also by ${ }^{*}$ used more commonly in rheology) is given by

$\tilde{\eta}(\omega)=\eta^{*}(\omega)=k_{B} T \frac{\left(\mathbf{M}_{\mathrm{St}}^{\mathrm{r}}\right)_{j k}}{\left\langle\mathcal{V}_{j}(0) \tilde{\mathcal{V}}_{k}(\omega)\right\rangle}$

Here, and in other such ratios, the Einstein summation convention is not used-instead, each ratio reflects one component of up to $N^{2}$ nontrivial equations inherent in the T-GSER. In principle, this tensorial expression of the GSER could potentially be used to determine $\tilde{\eta}(\omega)$ from up to 36 different non-zero elements in $\mathbf{M}_{\mathrm{St}}^{\mathrm{r}}$, if all components of the generalized velocity could be measured over a long enough duration to provide sufficient statistics. Since the complex shear modulus is $\tilde{G}=i \omega \tilde{\eta}$, it is possible to re-express Eq. 20 in a tensorial form of the GSER that is written using transformed MSDs rather than VCFs:

$\tilde{G}(\omega)=G^{*}(\omega)=\frac{2 k_{B} T\left(\mathbf{M}_{\mathrm{St}}^{\mathrm{r}}\right)_{j k}}{(i \omega)\left\langle\Delta \mathcal{R}_{j}(0) \Delta \tilde{\mathcal{R}}_{k}(\omega)\right\rangle}$.

Likewise, since $\tilde{G}(\omega) \tilde{J}(\omega)=(i \omega)^{-1}$, where $\tilde{J}$ is the transformed creep compliance (Bird et al. 1977), inverse transformation yields another equivalent expression for the tensorial GSER in terms of MSDs in the time domain. The creep compliance is simply proportional to the MSD (Xu et al. 1998):

$J(t)=\frac{\left\langle\Delta \mathcal{R}_{j}(0) \Delta \mathcal{R}_{k}(t)\right\rangle}{2 k_{B} T\left(\mathbf{M}_{\mathrm{St}}^{\mathrm{r}}\right)_{j k}}$.

Since $J$ in 3D has units of inverse energy density, the units of $\left\langle\Delta \mathcal{R}_{j} \Delta \mathcal{R}_{k}\right\rangle /\left(\mathbf{M}_{\mathrm{St}}^{\mathrm{r}}\right)_{j k}$ are always volume, regardless of whether the degrees of freedom corresponding to the chosen $j$ and $k$ are translational, rotational, or a combination thereof. The above three expressions for the tensorial GSER provide the material's LVE response in terms of the three most frequently used rheological properties, yet equivalent expressions for other properties (e.g., the stress relaxation modulus or retardation spectrum) could be derived.
In order to more precisely determine a material's LVE response, it is often desirable to measure more than one degree of freedom of a probe's motion. In a very simple example, three independent translational degrees of freedom of a sphere can be measured using 3D particle tracking methods. While it is possible to determine and report $\tilde{G}$ three separate times from each degree of freedom independently using Eq. 21, it is usually desirable to average the three results to provide a more precise value of the modulus. In fact, this averaging approach has been used since the first application of the GSER for spheres in a LVE material, and naturally occurs in dynamic light scattering measurements (Berne and Pecora 2000). Likewise, by determining $\tilde{G}$ independently many different times using multiple different components of the mobility tensor, statistical analysis of the set of functions $\{\tilde{G}(\omega)\}$ can potentially be used to isolate possible experimental biases in measuring one or more degrees of freedom.

Multi-particle tensorial GSER: grand mobility tensor

An additional generalization of the mobility tensor can be made when considering $N$ probe particles that interact in a LVE material. The translational and rotational velocities arising from the history of past forces and torques on each of the particles is given by:

$\left(\begin{array}{c}\mathbf{V}^{1}(t) \\ \boldsymbol{\Omega}^{1}(t) \\ \ldots \\ \mathbf{V}^{N}(t) \\ \boldsymbol{\Omega}^{N}(t)\end{array}\right)=\int_{-\infty}^{t} \mathcal{M}\left(t-t^{\prime}\right) \cdot\left(\begin{array}{c}\mathbf{F}^{1}\left(t^{\prime}\right) \\ \mathbf{T}^{1}\left(t^{\prime}\right) \\ \ldots \\ \mathbf{F}^{N}\left(t^{\prime}\right) \\ \mathbf{T}^{N}\left(t^{\prime}\right)\end{array}\right) \mathrm{d} t^{\prime}$

where

$\mathcal{M}(t)=\left(\begin{array}{ccc}\mathbf{M}^{11}(t) & \ldots & \mathbf{M}^{1 N}(t) \\ \ldots & \ldots & \ldots \\ \mathbf{M}^{N 1}(t) & \ldots & \mathbf{M}^{N N}(t)\end{array}\right)$

is the $6 N \times 6 N$ grand multi-particle mobility tensor, whose transform is the inverse of the transformed grand multiparticle resistance tensor:

$\tilde{\mathcal{M}}(\omega)=(\tilde{\mathcal{Z}}(\omega))^{-1}$

Diagonal blocks $\mathbf{M}^{K K}$ represent $6 \times 6$ self-mobility tensors, and off-diagonal blocks $\mathbf{M}^{K L}$ represent $6 \times 6$ coupling mobility tensors relating the translational and rotational velocities of particle $K$ to the forces and 
torques on particle $L$. To obtain all components of the grand multi-particle mobility tensor, it is necessary to perform a series of Stokes flow calculations to determine self- and coupling-mobility sub-tensors, using appropriate boundary conditions (e.g., no-slip boundary conditions).

The equations of motion for probe motion and fluid flow when calculating coupling mobility sub-tensors of $\tilde{\mathcal{M}}$ can become quite involved for all but the simpest geometries, and are analytically tractable only in the near- or a far-field approximations. Approximate formulae for the mobility tensor can be obtained when particles are well-separated, in which case the coupling mobilities (which relate the velocity of particle $L$ due to a force on particle $K$ ) through so-called reflection methods (Kim and Karilla 1991; Happel and Brenner 1983; Pozrikidis 1992). The far-field fluid flow velocity $\mathbf{v}$ resulting from a force on particle $K$ can be approximated by the flow due to a point force at the location of particle $K$. In a Newtonian Stokes flow, this flow is given by the Oseen tensor $\mathbf{S}$

$\mathbf{v}=\mathbf{S}\left(\mathbf{d}_{L}-\mathbf{d}_{K}\right) \cdot \mathbf{F}_{K}$, where $\mathbf{S}(\mathbf{d})=\frac{1}{8 \pi \eta_{0}}\left(\frac{\boldsymbol{\delta}}{d}+\frac{\mathbf{d d}}{d^{3}}\right)$,

and $d=|\mathbf{d}|=\left|\mathbf{d}_{L}-\mathbf{d}_{K}\right|$ represents the separation between the centers of self-mobility of particles $K$ and $L$. Reflection methods typically work when $d$ is significantly larger than the maximum spatial dimension of either particle. The leading-order approximation to the velocity of a particle in such a flow is given by Faxen's law to be simply the local fluid velocity at that position. Thus, the leading-order approximation to the (translational) coupling mobility $\mathbf{M}_{\mathbf{V} \mathbf{F}}^{K L}$ between particles $K$ and $L$ is the Oseen tensor. Kirkwood used this far-field approach to incorporating hydrodynamic interactions in bead-spring models of polymers, by using the Stokes self-mobility when $K=L$ and the Oseen tensor when $K \neq L$ (Kirkwood 1949); powerful extensions were given by Rotne and Prager, who added a short-range correction to make the mobility positive-definitive (Rotne and Prager 1969), and Brady \& Bossis, whose 'Stokesian Dynamics' method accurately and efficiently computes both far-field and near-field (lubrication) hydrodynamic interactions in many-body systems (Brady and Bossis 1988). The rotation of $K$ in response to a force on $L$ (coupling mobility $\mathbf{M}_{\Omega \mathbf{F}}^{K L}$ ) is given to leading order by the vorticity of the Oseen tensor. The coupling mobilities relating the translation $\mathbf{M}_{\mathbf{V T}}^{K L}$ and rotation $\mathbf{M}_{\Omega \mathbf{T}}^{K L}$ of $K$ in response to a torque on $L$ can be found using the flow due to a point torque (the rotlet), and so on. Using higher-order reflections, Batchelor explicitly computed the mobility tensor for two particles up to fourth order in the particle radii (Batchelor 1976), which Crocker measured directly using optical tweezers (Crocker 1997).

Thus, in principle, complex calculations of the full translational-rotational coupling between two anisotropic particles mediated by an LVE material can be performed, yielding tensorial expressions for twopoint microrheology in which the separation $\mathbf{d}$ appears explicitly and plays a prominent role. Under certain conditions, it may be possible to initially model the LVE material as a simple viscous liquid, simplifying the equations that must be solved to determine the Stokes coupling mobility, which could then be used to provide expressions of a tensorial two-point translationalrotational GSER. Thus, we have sketched the basic steps by which a tensorial two-point GSER that depends on d between two anisotropic particles can be obtained.

Multi-particle tensorial GSER: generalized

Langevin approach

In this section, we extend the derivation of a single particle tensorial GSER to include multiple particles, following a generalized Langevin approach that uses the grand multi-particle mobility tensor defined in the preceding section. As with a single anisotropic probe, the energy equipartition and fluctuation dissipation theorems provide the key simplifications. We start with the generalized Langevin equation for $N$ particles in a linear viscoelastic material, considering both translational and rotational degrees of freedom:

$$
\begin{gathered}
\left(\begin{array}{l}
m^{1} \dot{\mathbf{V}}^{1}(t) \\
I^{1} \dot{\mathbf{\Omega}}^{1}(t) \\
\cdots \\
m^{N} \dot{\mathbf{V}}^{N}(t) \\
I^{N} \dot{\boldsymbol{\Omega}}^{N}(t)
\end{array}\right)=\left(\begin{array}{c}
\mathbf{f}_{R}^{1}(t) \\
\mathbf{T}_{R}^{1}(t) \\
\ldots \\
\mathbf{f}_{R}^{N}(t) \\
\mathbf{T}_{R}^{N}(t)
\end{array}\right) \\
-\int_{-\infty}^{t} \mathcal{Z}\left(t-t^{\prime}\right) \cdot\left(\begin{array}{c}
\mathbf{V}^{1}\left(t^{\prime}\right) \\
\mathbf{\Omega}^{1}\left(t^{\prime}\right) \\
\ldots \\
\mathbf{V}^{N}\left(t^{\prime}\right) \\
\mathbf{\Omega}^{N}\left(t^{\prime}\right)
\end{array}\right) \mathrm{d} t^{\prime},
\end{gathered}
$$

where $\mathbf{f}_{R}^{K}(t)$ and $\mathbf{T}_{R}^{K}(t)$ are the stochastic thermal force and torque, respectively, on particle $K$. Equation 27 represents a balance of forces on the particles, with changes in translational and rotational inertia of the 
particles balanced by the forces and torques on the particles moving within the material due to hydrodynamic stresses as well as stochastic (thermal) collisions. In tensor form, Eq. 27 reduces to

$\mathbb{M} \cdot \dot{\mathcal{V}}(t)=\mathcal{F}_{R}(t)-\int_{-\infty}^{t} \mathcal{Z}\left(t-t^{\prime}\right) \cdot \mathcal{V}\left(t^{\prime}\right) \mathrm{d} t^{\prime}$,

where $\mathbb{M}$ is a diagonal $6 N \times 6 N$ grand multi-particle inertia tensor populated with appropriate masses and moments of inertia, and $\mathcal{F}_{R}(t)=\left\{\mathbf{f}_{R}^{1}, \mathbf{T}_{R}^{1}, \ldots, \mathbf{f}_{R}^{N}, \mathbf{T}_{R}^{N}\right\}$ is a vector of $6 \mathrm{~N}$ components expressing random forces and torques on the respective particles. The unilateral Fourier transform of Eq. 28 can be solved for the $k$ th component of $\tilde{\mathcal{V}}$ :

$$
\tilde{\mathcal{V}}_{k}(\omega)=(i \mathbb{M} \omega+\tilde{\mathcal{Z}}(\omega))_{k \ell}^{-1} \cdot\left(\tilde{\mathcal{F}}_{R}(\omega)+\mathbb{M} \cdot \mathcal{V}(0)\right)_{\ell}
$$

Following the same steps as for the single anisotropic probe case, we calculate the transformed VCF and its related MSD in terms of the inverse of the grand resistance tensor, using statistical independence $\left\langle\mathcal{V}_{j} \mathcal{F}_{R, \ell}\right\rangle=0$ and energy equipartition $\left\langle\mathcal{V}_{j}(0)(\mathbb{M}\right.$. $\left.\mathcal{V}(0))_{\ell}\right\rangle=k_{B} T \delta_{j \ell}$, giving:

$$
\begin{aligned}
\left\langle\mathcal{V}_{j}(0) \tilde{\mathcal{V}}_{k}(\omega)\right\rangle & =\frac{(i \omega)^{2}}{2} \mathcal{F}^{u}\left\langle\Delta \mathcal{R}_{j}(0) \Delta \mathcal{R}_{k}(t)\right\rangle \\
& =k_{B} T(i \mathbb{M} \omega+\tilde{\mathcal{Z}}(\omega))_{j k}^{-1} .
\end{aligned}
$$

When the LVE resistance $|\tilde{\mathcal{Z}}(\omega)|$ dominates over probe inertia $|\mathbb{M} \omega|$, this simplifies to

$$
\begin{aligned}
\left\langle\mathcal{V}_{j}(0) \tilde{\mathcal{V}}_{k}(\omega)\right\rangle & =\frac{(i \omega)^{2}}{2}\left\langle\Delta \mathcal{R}_{j}(0) \Delta \tilde{\mathcal{R}}_{k}(\omega)\right\rangle \\
& =k_{B} T(\tilde{\mathcal{Z}}(\omega))_{j k}^{-1}=k_{B} T(\tilde{\mathcal{M}}(\omega))_{j k}
\end{aligned}
$$

A microrheological measurement of the (transformed) correlation between probe displacements in two 'directions' $\Delta \mathcal{R}_{j}$ and $\Delta \mathcal{R}_{k}$, then, is proportional to the $j k$ th component of the (transformed) grand multi-particle mobility tensor $\tilde{\mathcal{M}}$. This result, which essentially expresses the Einstein component of the GSER, holds for translation and rotation, for the autocorrelation of a single probe or the cross-correlation of two probes (Levine and Lubensky 2000; Crocker et al. 2000).

The generalization of the Stokes mobility to cover a wide frequency range can be made for the grand multiparticle mobility tensor. Essentially this generalization assumes that frequency-dependence of $\tilde{\mathcal{M}}(\omega)$ arises only from the complex viscosity of the LVE material:

$\tilde{\mathcal{M}}(\omega)=\frac{\eta_{0}}{\tilde{\eta}(\omega)} \mathcal{M}_{\mathrm{St}}$

where $\mathcal{M}_{\mathrm{St}}^{\mathrm{r}}=\eta_{0} \mathcal{M}_{\mathrm{St}}$ as in the single particle case. The existence of a separation distance $d$ places more severe restrictions on the quasi-steady assumption inherent in the standard GSM, and the effects of material inertia have been directly measured in cross-correlation measurements (Atakhorrami et al. 2005, 2008).

Combining the Einstein and Stokes components of the multi-particle results, equivalent statements of the GSER for multiple anisotropic particles can be made as follows. The complex viscosity is given by thermal energy times the ratio of a scalar component of the reduced Stokes mobility tensor and corresponding VCF:

$\tilde{\eta}(\omega)=\eta^{*}(\omega)=k_{B} T \frac{\left(\mathcal{M}_{\mathrm{St}}^{\mathrm{r}}\right)_{j k}}{\left\langle\mathcal{V}_{j}(0) \tilde{\mathcal{V}}_{k}(\omega)\right\rangle}$,

the complex shear modulus can be expressed in terms of transformed MSDs:

$\tilde{G}(\omega)=G^{*}(\omega)=\frac{2 k_{B} T\left(\mathcal{M}_{\mathrm{St}}^{\mathrm{r}}\right)_{j k}}{(i \omega)\left\langle\Delta \mathcal{R}_{j}(0) \Delta \tilde{\mathcal{R}}_{k}(\omega)\right\rangle}$,

and the creep compliance is proportional to the various time-domain MSDs:

$J(t)=\frac{\left\langle\Delta \mathcal{R}_{j}(0) \Delta \mathcal{R}_{k}(t)\right\rangle}{2 k_{B} T\left(\mathcal{M}_{\mathrm{St}}^{\mathrm{r}}\right)_{j k}}$.

Again, no summation is implied despite the repeated $j k$ indices on the right-hand side. Embodied in these compact expressions of the multi-particle GSER in Eqs. 33-35 is the potential to use a large number of degrees of translational and rotational freedom of many probe particles to extract the LVE rheology of a material with high precision through averaging.

\section{Reduced Stokes self-mobility tensors for bodies of revolution}

We now provide several concrete examples of reduced Stokes self-resistance and self-mobility tensors for probes having shapes that are experimentally realizable, yet also having anisotropies that impart nontrivial features to their dynamics. As detailed in various texts (Happel and Brenner 1983; Kim and Karilla 1991; Leal 2007), the linear response of a general particle subject 
to a specified rotation and translation in Stokes flow, and vice versa, expressed in terms of the reduced Stokes resistance tensor is:

$$
\left(\begin{array}{c}
\mathbf{F} \\
\mathbf{T}
\end{array}\right)=\eta_{0} \zeta_{\mathbf{S t}}^{\mathbf{r}} \cdot\left(\begin{array}{l}
\mathbf{V} \\
\boldsymbol{\Omega}
\end{array}\right)=\eta_{0}\left(\begin{array}{ll}
\mathbf{A} & \mathbf{B}^{T} \\
\mathbf{B} & \mathbf{C}
\end{array}\right) \cdot\left(\begin{array}{l}
\mathbf{V} \\
\boldsymbol{\Omega}
\end{array}\right)
$$

and equivalently expressed in terms of the reduced Stokes mobility tensor is:

$$
\left(\begin{array}{l}
\mathbf{V} \\
\boldsymbol{\Omega}
\end{array}\right)=\eta_{0}^{-1} \mathbf{M}_{\mathrm{St}}^{\mathrm{r}} \cdot\left(\begin{array}{c}
\mathbf{F} \\
\mathbf{T}
\end{array}\right)=\eta_{0}^{-1}\left(\begin{array}{ll}
\mathbf{D} & \mathbf{E}^{T} \\
\mathbf{E} & \mathbf{H}
\end{array}\right) \cdot\left(\begin{array}{l}
\mathbf{F} \\
\mathbf{T}
\end{array}\right)
$$

Comparison with Eq. 6 shows that the sub-blocks can be related to the reduced Stokes mobilities: $\left(\mathbf{M}_{\mathrm{St}}^{\mathrm{r}}\right)_{\mathbf{V F}}=\mathbf{D}$, $\left(\mathbf{M}_{\mathrm{St}}^{\mathrm{r}}\right)_{\mathbf{V T}}=\mathbf{E}^{T},\left(\mathbf{M}_{\mathrm{St}}^{\mathrm{r}}\right)_{\boldsymbol{\Omega} F}=\mathbf{E}$, and $\left(\mathbf{M}_{\mathrm{St}}^{\mathrm{r}}\right)_{\boldsymbol{\Omega} \mathbf{T}}=\mathbf{H}$. Here, we will restrict our discussion to bodies of revolution about a symmetry axis $\mathbf{p}$, for which the $3 \times 3$ sub-blocks of the resistance tensor are $\mathbf{A}$ and $\mathbf{C}$. These have the form (Kim and Karilla 1991):

$$
\begin{aligned}
& \mathbf{A}_{j k}=X^{A} p_{j} p_{k}+Y^{A}\left(\delta_{j k}-p_{j} p_{k}\right) \\
& \mathbf{C}_{j k}=X^{C} p_{j} p_{k}+Y^{C}\left(\delta_{j k}-p_{j} p_{k}\right),
\end{aligned}
$$

and the $3 \times 3$ sub-block $\mathbf{B}$ is an anti-symmetric tensor of the form:

$\mathbf{B}_{j k}=-\mathbf{B}_{k j}=Y^{B} \epsilon_{j k l} p_{l}$.

Here $\epsilon_{j k l}$ is the usual completely antisymmetric thirdrank tensor, which is +1 for $\{j k l\}=\{123\}$ and switches sign when any pair of indices is reversed. The subblocks of the mobility tensor are, in turn, given by:

$$
\begin{aligned}
\mathbf{D}_{j k} & =\left(X^{A}\right)^{-1} p_{j} p_{k}-\alpha Y^{C}\left(\delta_{j k}-p_{j} p_{k}\right) \\
\mathbf{E}_{j k} & =-\mathbf{E}_{k j}=\alpha Y^{B} \epsilon_{j k l} p_{l} . \\
\mathbf{H}_{j k} & =\left(X^{C}\right)^{-1} p_{j} p_{k}-\alpha Y^{A}\left(\delta_{j k}-p_{j} p_{k}\right),
\end{aligned}
$$

where $\alpha=\left[\left(Y^{B}\right)^{2}-Y^{A} Y^{C}\right]^{-1}$. If the symmetry axis $\mathbf{p}$ of the particle is aligned in the 1-direction, explicit expressions for the resistance and mobility tensors are:

$$
\zeta_{\mathrm{St}}^{\mathrm{r}}=\left(\begin{array}{cccccc}
X^{A} & 0 & 0 & 0 & 0 & 0 \\
0 & Y^{A} & 0 & 0 & 0 & -Y^{B} \\
0 & 0 & Y^{A} & 0 & Y^{B} & 0 \\
0 & 0 & 0 & X^{C} & 0 & 0 \\
0 & 0 & Y^{B} & 0 & Y^{C} & 0 \\
0 & -Y^{B} & 0 & 0 & 0 & Y^{C}
\end{array}\right)
$$

$$
\mathbf{M}_{\mathrm{St}}^{\mathrm{r}}=\left(\begin{array}{cccccc}
\left(X^{A}\right)^{-1} & 0 & 0 & 0 & 0 & 0 \\
0 & -\alpha Y^{C} & 0 & 0 & 0 & -\alpha Y^{B} \\
0 & 0 & -\alpha Y^{C} & 0 & \alpha Y^{B} & 0 \\
0 & 0 & 0 & \left(X^{C}\right)^{-1} & 0 & 0 \\
0 & 0 & \alpha Y^{B} & 0 & -\alpha Y^{A} & 0 \\
0 & -\alpha Y^{B} & 0 & 0 & 0 & -\alpha Y^{A}
\end{array}\right) .
$$

The coefficients $X^{A, C}$ and $Y^{A, B, C}$ depend upon the nature (e.g., geometry and material) of the specific system considered. Expressions for a variety of shapes in Newtonian Stokes flows have been computed and tabulated in the literature (e.g., Kim and Karilla 1991; Happel and Brenner 1983). Such reduced Stokes mobility tensors can be used directly to give the microrheological response in linear viscoelastic materials; we give below several examples of particular interest.

Ellipsoids of revolution, or spheroids, which obey

$\frac{x^{2}}{c^{2}}+\frac{y^{2}}{c^{2}}+\frac{z^{2}}{a^{2}}=1$,

can be either prolate $(a>c)$ or oblate $(a<c)$. The eccentricity of a prolate spheroid is given by $e_{p}=$ $\sqrt{1-(c / a)^{2}}$, whereas that of on oblate spheroid is $e_{o}=$ $\sqrt{(a / c)^{2}-1}$. Their resistance functions are given in Table 1. Notably, the resistance (both to translation and rotation) each have two distinct values - one for motion along the symmetry axis $\mathbf{p}$, and another for motion perpendicular to $\mathbf{p}$. Due to the fore-aft symmetry of ellipsoids, there is no translation-rotation coupling, and $\mathbf{B}=0$.

Bodies of revolution without fore-aft symmetry, on the other hand, will generally exhibit translationrotation coupling. Nir and Acrivos (1973), for example, computed the resistance tensor for anisotropic 'dumbbell' particles composed of two spheres of arbitrary radii in contact with each other. When the sphere radii are different, $Y^{B}$ (and thus $\mathbf{B}$ ) is generally non-zero; computed values for various radius ratios are given in Nir and Acrivos (1973). A simpler system, for which approximate formulae exist, was explored by Ramachandran and Khair (2009): a 'Janus' sphere consisting of a 'no-slip' portion and a portion with slight solid-liquid slip, with slip length $\lambda_{s}$ which was assumed small compared with the particle radius $a$. In Table 1, we quote the resistance for a sphere that is exactly half 'stick' and half 'slip', and note that more general geometries are treated in Ramachandran and Khair (2009). As with fore-aft symmetric bodies of rotation, symmetries demand two distinct values for the resistance, corresponding to translation or rotation parallel and perpendicular to $\mathbf{p}$. On the other hand, fore-aft asymmetric bodies, 
Table 1 Coefficients for resistance and mobility matrices (Eqs. 38-43) for several axisymmetric bodies

\begin{tabular}{|c|c|c|c|}
\hline & Prolate spheroid & Oblate spheroid & $\begin{array}{l}\text { Janus sphere } \\
\text { (half-stick, half-slip) }\end{array}$ \\
\hline$X^{A}$ & $\frac{12 \pi a e_{p}^{3}}{-2 e_{p}+\left(1+e_{p}^{2}\right) L}$ & $\frac{8 \pi a e_{o}^{3}}{\left(2 e_{o}^{2}-1\right) C+e_{o} \sqrt{1-e_{o}^{2}}}$ & $6 \pi a\left(1-\frac{\beta}{2}\right)$ \\
\hline$X^{C}$ & $\frac{32 \pi a^{3} e_{p}^{3}\left(1-e_{p}^{2}\right)}{3\left(2 e_{p}-\left(1-e_{p}^{2}\right) L\right)}$ & $\frac{16 \pi a^{3} e_{o}^{3}}{3\left(C-e_{o} \sqrt{1-e_{o}^{2}}\right)}$ & $8 \pi a^{3}\left(1-\frac{3 \beta}{2}\right)$ \\
\hline$Y^{A}$ & $\frac{32 \pi a e_{p}^{3}}{2 e_{p}+\left(3 e_{p}^{2}-1\right) L}$ & $\frac{16 \pi a e_{o}^{3}}{\left(2 e_{o}^{2}+1\right) C-e_{o} \sqrt{1-e_{o}^{2}}}$ & $6 \pi a\left(1-\frac{\beta}{2}\right)$ \\
\hline$Y^{B}$ & 0 & 0 & $\frac{9}{2} \beta a^{2}$ \\
\hline$Y^{C}$ & $\frac{32 \pi a^{3} e_{p}^{2}\left(2-e_{p}^{2}\right)}{3\left(-2 e_{p}+\left(1+e_{p}^{2}\right) L\right)}$ & $\frac{16 \pi a^{3} e_{o}^{3}\left(2-e_{o}^{2}\right)}{3\left(e_{o} \sqrt{1-e_{o}^{2}}-\left(1-2 e_{o}^{2}\right) C\right)}$ & $8 \pi a^{3}\left(1-\frac{3 \beta}{2}\right)$ \\
\hline Where & $L\left(e_{p}\right)=\ln \left(\frac{1+e_{p}}{1-e_{p}}\right)$ & $C\left(e_{o}\right)=\cot ^{-1}\left(\frac{\sqrt{1-e_{o}^{2}}}{e_{o}}\right)$ & $\beta=\frac{\lambda_{s}}{a} \ll 1$ \\
\hline
\end{tabular}

The fore-aft symmetry of the spheroids results in zero translation-rotation coupling $\left(Y^{B}=0\right)$, whereas the fore-aft asymmetric Janus spheres and asymmetric dumbbells of Nir and Acrivos (1973), have a non-zero translation-rotation coupling $\left(Y^{B}\right)$

including the Janus sphere, exhibit translation-rotation coupling. Breaking rotational symmetry-e.g., particles with chirality-introduces further richness into the resistance and mobility tensors; a potential example for such a probe is a chiral colloidal aggregate (Keaveny and Shelley 2009).

These represent but a few examples of the rich dynamics that are exhibited by probes that progressively break the (isotropic) symmetry of spheres. As shown above, one can easily generalize the Stokes resistance tensors for such probes in Newtonian fluids to describe probe motion in linear viscoelastic materials, in a manner precisely analogous to the standard GSER used for passive microrheology.

\section{Discussion}

Beyond generalizing the Stokes-Einstein relation to the frequency domain, the preceding theoretical treatment further extends this key relation to cover multiple degrees of translational and rotational freedom for one or more probes that can have anisotropic shapes. Advanced optical microscopy techniques, including particle tracking in three dimensions, may make it feasible to measure 3-d translational and rotational trajectories and compute the spatially-dependent LVE properties of a complex fluid based on the multi-particle, multi-DOF GSER. This is an interesting direction in microrheology, since the combined translational and rotational motion of even a single anisotropic probe particle has not yet been measured simultaneously in 3d. Although the amount of data that must be processed and averaged to calculate every possible VCF or MSD for all potential degrees of freedom may be vast and has been typically treated up to now through postacquisition processing, advances in computing technology and software may make it feasible in the future to calculate LVE rheology from the dynamics of multiple proximate anisotropic probes in real-time.

For simple, isotropic, incompressible LVE materials, since each probe DOF encodes the same material property $G^{*}(\omega)$, tracking multiple degrees of freedom effectively provides a self-consistency check and also additional statistics, rather than access to fundamentally new information about material properties. Indeed, additional statistics of the translational motion of spherical probes measured in two or three dimensions has been inherent in the common implementations of the GSER since the advent of passive microrheology. If, however, the measured moduli determined from the various DOFs of the same probe are not self-consistent, then it is possible that the material violates one of the assumptions required for the generalized Stokes mobility to hold (Squires, in preparation). In that case, independent measurements using different DOFs of the same probe can provide additional information about the material or about the coupling of the material to the probe. For example, a spherical probe in a nematic liquid crystal (Loudet et al. 2004; Stark 2001) exhibits 
an anisotropic diffusivity, which has different values when the sphere's motion is parallel with or perpendicular to the local director field. From symmetry alone, one would expect two distinct translational diffusivities and two distinct rotational diffusivities; however, five distinct viscosities are generally required for a complete material description of a nematic liquid crystal (Stark 2001). By contrast, an ellipsoidal probe, when oriented perpendicular to the local director, can translate in three distinct directions and, when oriented parallel with the director, can translate in two distinct directions, thus giving five independent measurements and thereby enabling the five independent viscosities, in principle, to be recovered. The rotational diffusion of ellipsoidal probes, then, would in principle give redundant information, and could be used as a check or to probe further aspects of the material (e.g., compressibility or defects).

Additionally, some materials (e.g., networks of semiflexible polymers in solution (Chen et al. 2003)) are known to form depletion zones having lower concentrations of elastic supramolecular structures around probes. In addition to providing an inhomogeneously 'soft' local environment that modifies the translational mobility (Levine and Lubensky 2000, 2001), a depletion zone can also effectively decouple the elastic network from the probe's surface. The effective slip exhibited by the reduction in local concentration of elastic components near the probe's boundary affects the rotational mobility much more significantly than translation (Schmiedeberg and Stark 2005), since an axisymmetric probe can slowly rotate about its axis without significantly deforming the elastic phase outsize the depletion zone. For example, comparing the rotational diffusion of an ellipsoid about its axis of symmetry with either its translation or its non-axisymmetric rotation could provide a quantitative means of probing the nature and extent of the depletion zone. On the other hand, more geometrically complex probes may not be able to rotate without physically forcing the network to deform, irrespective of how easily the network may appear to slip.

Finally, some soft materials-polymer solutions, for example - can contain a sparse dispersion of a compressible elastic phase (Poisson ratio $v \neq 1 / 2$ ) within an incompressible fluid. Under steady or low-frequency forcing, the elastic structures compress, squeezing fluid into and out of regions of the material as required to maintain fluid incompressibility. The coupling of the fluid phase to the elastic phase weakens near a time scale $\tau_{c}$, estimated for polymers by Gittes et al. (1997), that is set by balancing viscous stresses $\eta U / \xi^{2} \tau_{c}$ (e.g., though pores in the elastic phase which have a char- acteristic length scale $\xi$, where $U$ is the displacement relative to the network) against elastic stresses in the material $G U / R_{d}^{2}$, where $R_{d}$ is the length scale over which the deformations occur. For spherical probes, this gives the frequency $\omega_{c} \sim G \xi^{2} / \eta R_{d}^{2}$ below which the viscous phase is effectively decoupled from the elastic one, causing a violation of the assumption of incompressibility inherent in the GSER. Thus, for this case in which compressibility cannot be neglected, if the translational and rotational diffusivities of a spherical probe were compared, one would anticipate quantitative agreement at high frequencies $\left(\omega \gg \omega_{c}\right)$, assuming no depletion region or slip layer forms around the probe; however, it is likely that a significant discrepancy would be found at low frequencies. For ellipsoidal probes, axisymmetric rotations force pure shear deformations, and therefore would not compress or dilate the material, whereas translational motion would not create pure shear deformations. By contrast, non-axisymmetric rotations of ellipsoidal probes would drive both shear and compression within a compressible material. Thus, for geometrically complex colloids, if different modes of motion yield results for moduli that appear to be inconsistent, one can potentially extract additional information about material inhomogeneity or about the coupling of the probe to the material that would have been inaccessible using only standard spherical probes.

Since elements of the reduced Stokes mobility tensors are typically referenced relative to a specific instantaneous orientation of anisotropic probe particles (e.g., a symmetry axis in the case of spheroids), in experiments, it can be important to calculate VCFs and MSDs using appropriate coordinate systems that reflect probe orientation. Because probe displacements and rotations are typically not very large in passive microrheology, it may be possible to ignore changes in probe orientation in some cases. However, for LVE materials that exhibit long-time dissipative relaxations, it may be necessary to create a smooth temporally averaged orientation and compute relative VCFs and MSDs of translational and rotational motion based on fluctuations relative to this slow-moving average in order to properly apply the tensorial GSER using appropriate elements of the reduced Stokes mobility tensor. For instance, the parallel and perpendicular mobilities of spheroids are typically referenced relative to an instantaneous orientation of the symmetry axis, yielding expressions for parallel and perpendicular translational and rotational diffusion coefficients. Also, for spheroids, unless a particle is specially labeled, it is usually quite difficult to distinguish and measure rotational motion about its symmetry axis, whereas it can be much easier to measure the rotation of the symmetry 
axis itself through methods such as real-space tracking (Cheng and Mason 2003; Han et al. 2006) or light streak tracking (Cheng et al. 2002; Cheng and Mason 2003).

Since the Stokes flow problem has only been solved to provide the complete Stokes mobility tensors for some of the simplest shapes, the extension of the GSER to anisotropic probes highlights the need to compute these tensors for more complicated shapes. In many cases, the form of the tensors will be simply diagonal, but in the case of chiral particles, off-diagonal elements could play an interesting role, and there is a potential to determine LVE properties from translationalrotational coupling dynamics of chiral probes (e.g., Keaveny and Shelley 2009).

Anisotropic probes could provide certain advantages for exploring the LVE properties of materials that have inhomogeneous molecular structure and anisotropy, such as in liquid crystals. The generalized Langevin equations of motion for the probes would necessarily be even more complex than those we have written for the simplest case of an isotropic LVE material. In many cases, it would be necessary to solve these equations of motion self-consistently with the equations of motion for the director field of the anisotropic molecular liquid crystal. In fact, recent experiments have shown that attractive interactions can exist between neighboring proximate anisotropic probes even in simple nematic liquid crystals (Lapointe et al. 2009; Stark 2001), and these attractions can depend upon the particular type of anchoring of the liquid crystal, and the relative positions and orientations of the probe particles. Such higher levels of complexity introduced by anisotropic materials are well beyond the scope of the treatment we have presented, and they offer an important challenge for future theory in the field of microrheology.

\section{Conclusion}

This work provides the basic theoretical foundation for extending the generalized Stokes-Einstein relation to handle translation and rotation of an anisotropic probe in an isotropic LVE material, as well as to treat twopoint microrheology of multiple anisotropic probes. The basic relationships for the T-GSER rely upon the same fundamental physics of the energy equipartition and fluctuation-dissipation theorems that are contained in the simplest original expression of the GSER. Therefore, it is not surprising that the form of the T-GSER is quite similar to the original expressions, and the LVE rheology of the material can be extracted from one or more of many possible degrees of freedom. Averaging results of the LVE properties over multiple degrees of freedom of one or more probe particles using the TGSER can provide a more precise and accurate value of the frequency-dependent linear viscoelasticity of complex fluids. We anticipate that this work will motivate both future calculations of Stokes mobility tensors and also experiments that determine linear viscoelasticity from translational-rotational coupling of chiral probe particles using the T-GSER. As new methods of colloidal synthesis are producing many different kinds of geometrically complex anisotropic particles, this work provides a context for interpreting future microrheology experiments that use complex shaped particles as probes in viscoelastic materials.

Acknowledgements T.M.S. gratefully acknowledges support from the National Science Foundation (CBET 730270).

Open Access This article is distributed under the terms of the Creative Commons Attribution Noncommercial License which permits any noncommercial use, distribution, and reproduction in any medium, provided the original author(s) and source are credited.

\section{References}

Atakhorrami M, Koenderink GH, Schmidt CF, MacKintosh FC (2005) Short-time inertial response of viscoelastic fluids: observation of vortex propagation. Phys Rev Lett 95(20):208302

Atakhorrami M, Mizuno D, Koenderink GH, Liverpool TB, MacKintosh FC, Schmidt CF (2008) Short-time inertial response of viscoelastic fluids measured with Brownian motion and with active probes. Phys Rev E 77(6):061508

Andablo-Reyes E, Diaz-Leyva P, Arauz-Lara JL (2005) Microrheology from rotational diffusion of colloidal particles. Phys Rev Lett 94:106001

Badaire S, Cottin-Bizonne C, Woody JW, Yang A, Stroock AD (2007) Shape selectivity in the assembly of lithographically designed colloidal particles. J Am Chem Soc 129(1):40-41

Batchelor GK (1976) Brownian diffusion of particles with hydrodynamic interaction. J Fluid Mech 74:1-29

Berne BJ, Pecora R (2000) Dynamic light scattering. Dover, New York

Bishop AI, Nieminen TA, Heckenberg NR, Rubinsztein-Dunlop $\mathrm{H}$ (2004) Optical microrheology using rotating laser-trapped particles. Phys Rev Lett 92(19):198104

Brady JF, Bossis G (1988) Stokesian dynamics. Annu Rev Fluid Mech 20:111-157

Breedveld V, Pine DJ (2003) Microrheology as a tool for highthroughput screening. J Mater Sci 38(22):4461-4470

Bird RB, Armstrong RC, Hassager O (1977) Dynamics of polymeric liquids. Wiley, New York

Champion JA, Katare YK, Mitragotri S (2007) Making polymeric micro- and nanoparticles of complex shapes. Proc Natl Acad Sci USA 104(29):11901-11904

Chen DT, Weeks ER, Crocker JC, Islam MF, Verma R, Gruber J, Levine AJ, Lubensky TC, Yodh AG (2003) Rheological microscopy: local mechanical properties from microrheology. Phys Rev Lett 90(10):108301 
Cheng Z, Mason TG (2003) Rotational diffusion microrheology. Phys Rev Lett 90(1):018304

Cheng Z, Chaikin PM, Mason TG (2002) Light streak tracking of optically trapped thin microdisks. Phys Rev Lett 89(1):108303

Crocker JC (1997) Measurement of the hydrodynamic corrections to the Brownian motion of two colloidal spheres. J Chem Phys 106(7):2837-2840

Crocker JC, Valentine MT, Weeks ER, Gisler T, Kaplan PD, Yodh AG, Weitz DA (2000) Two-point microrheology of inhomogeneous soft materials. Phys Rev Lett 85(4):888891

Davies B (2002) Integral transforms and their applications, 3rd edn. Springer, New York (2002)

Gardel ML, Weitz DA (2005) Microrheology. In: Breuer KS (ed) Microscale diagnostic techniques. Springer, Berlin, pp 1-50

Gittes F, Schnurr B, Olmsted PD, MacKintosh FC, Schmidt CF (1997) Microscopic viscoelasticity: shear moduli of soft materials determined from thermal fluctuations. Phys Rev Lett 79(17):3286-3289

Han Y, Alsayed A, Nobili M, Zhang J, Lubensky TC, Yodh AG (2006) Brownian motion of an ellipsoid. Science 314(1):626630

Han Y, Alsayed A, Nobili M, Yodh AG (2009) Quasi-twodimensional diffusion of single ellipsoids: aspect ratio and confinement effects. Phys Rev E 80(1):011403

Happel J, Brenner H (1983) Low Reynolds number hydrodynamics. Martinus Nijhoff, The Hague

Hernandez CJ, Mason TG (2007) Colloidal alphabet soup: monodisperse dispersions of shape-designed lithoparticles. J Phys Chem C 111(1):4477-4480

Keaveny EE, Shelley MJ (2009) Hydrodynamic mobility of chiral colloidal aggregates. Phys Rev E 79(5):051405.

Kim S, Karilla SJ (1991) Microhydrodynamics: principles and selected applications. Butterworth-Heinemann, Boston

Kirkwood JG (1949) The statistical mechanical theory of irreversible processes in solutions of flexible macromoleculesvisco-elastic behavior. Recl Trav Chim Pays-Bas (Journal of the Royal Netherlands Chemical Society) 68(9-10):649-660

Landau LD, Lifshitz EM (2000) Statistical physics. ButterworthHeinemann, Oxford

Lapointe C, Mason TG, Smalyukh II (2009) Shape-controlled colloidal interactions in a nematic liquid crystal. Science 326(5956):1083-1086

Leal LG (2007) Advanced transport phenomena: fluid mechanics and convective transport processes. Cambridge University Press, Cambridge

Levine AJ, Lubensky TC (2000) One- and two-particle microrheology. Phys Rev Lett 85(8):1774-1777

Levine AJ, Lubensky TC (2001) Response function of a sphere in a viscoelastic two-fluid medium. Phys Rev E 6304(4):041510

Loudet JC, Hanusse P, Poulin P (2004) Stokes drag on a sphere in a nematic liquid crystal. Science 306(5701):1525-1525
Manoharan VN, Elsesser MT, Pine DJ (2003) Dense packing and symmetry in small clusters of microspheres. Science 301(1):483-487

Mason TG (2000) Estimating the viscoelastic moduli of complex fluids using the generalized Stokes-Einstein equation. Rheol Acta 39(4):371-378

Mason TG (2002) Osmotically driven shape-dependent colloidal separations. Phys Rev E 66(1):060402(R)

Mason TG, Weitz DA (1995) Optical measurements of frequencydependent linear viscoelastic moduli of complex fluids. Phys Rev Lett 74(7):1250-1253

Mason TG, Ganesan K, vanZanten JH, Wirtz D, Kuo SC (1997a) Particle tracking microrheology of complex fluids. Phys Rev Lett 79(17):3282-3285

Mason TG, Gang H, Weitz DA (1997b) Optical measurements of the linear viscoelastic moduli of complex fluids. J Opt Soc Am A 14(1):139-149

Mizuno D, Tardin C, Schmidt CF, MacKintosh FC (2007) Nonequilibrium mechanics of active cytoskeletal networks. Science 315(5810):370-373

Nir A, Acrivos A (1973) Creeping motion of 2 arbitrary-sized touching spheres in a linear shear field. J Fluid Mech 59(Jun 19):209-223

Pozrikidis C (1992) Boundary integral and singularity methods for linearized viscous flow. Cambridge University Press, Cambridge

Ramachandran A, Khair AS (2009) The dynamics and rheology of a dilute suspension of hydrodynamically janus spheres in a linear flow. J Fluid Mech 633:233-269

Reif F (1965) Fundamentals of statistical and thermal physics. McGraw-Hill

Roos BW (1969) Analytic functions and distributions in physics and engineering. Wiley, New York

Rotne J, Prager S (1969) Variational treatment of hydrodynamic interaction in polymers. J Chem Phys 50(11):4831

Sato J, Breedveld V (2006) Transient rheology of solventresponsive complex fluids by integrating microrheology and microfluidics. J Rheol 50(1):1-19

Schmiedeberg M, Stark H (2005) One-bead microrheology with rotating particles. Europhys Lett 69(4):629-635

Schnurr B, Gittes F, MacKintosh FC, Schmidt CF (1997) Determining microscopic viscoelasticity in flexible and semiflexible polymer networks from thermal fluctuations. Macromolecules 30(25):7781-7792

Squires TM, Mason TG (2010) The fluid mechanics of microrheology. Annu Rev Fluid Mech 42:413-438

Stark H (2001) Physics of colloidal dispersions in nematic liquid crystals. Phys Rep-Rev Sec Phys Lett 351(6):387-474

Xu JY, Viasnoff V, Wirtz D (1998) Compliance of actin filament networks measured by particle-tracking microrheology and diffusing wave spectroscopy. Rheol Acta 37(4):387-398

Zwanzig R, Bixon M (1970) Hydrodynamic theory of velocity correlation function. Phys Rev A 2(5):2005-2012 